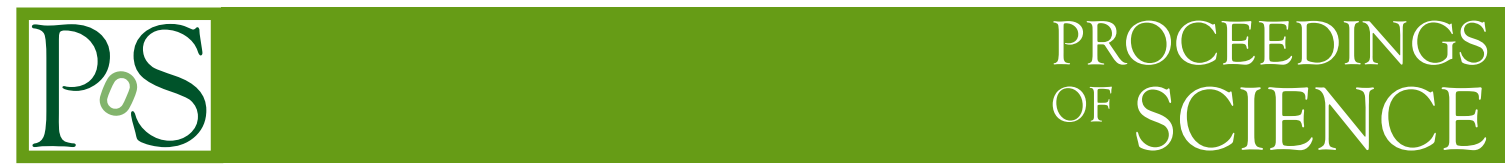

\title{
Witten parameter in pure gauge SU(2) theory
}

\author{
Vladimir Goy and Alexander Molochkov* \\ School of Biomedicine, Far Eastern Federal University \\ E-mail: vovagoy@gmail.com, molochkov.alexander@gmail.com
}

\begin{abstract}
The surface operator in an $S U(2)$ non-Abelian gauge field theory is studied. We analyse abelian projection of the $S U(2)$ symmetry to the $U(1)$ group calculating the Witten parameter using multilevel and multi-hit algorithms for the sake of statistical confidence. It is demonstrated that the Witten parameter depends on the surface area and volume in both phases.
\end{abstract}

Xth Quark Confinement and the Hadron Spectrum,

October 8-12, 2012

TUM Campus Garching, Munich, Germany

${ }^{*}$ Speaker. 


\section{Introduction}

In the four-dimensional gauge filed theory the most important probes for the phase states are the Wilson and t'Hooft line operatros that are difined on one-dimensional curves in the space-time. For example, these line-operators define order parameters for the confinemnet-deconfiment phase transition of the QCD vacuum. However, for more detail understanding of four-dimensional gauge field theory dynamics we need additional probes expressed by operators defined on the subspaces with higher dimensions. Possible candidates are operators that are defined on the two-dimensional surface in the four-dimensional space-time.

As an example we consider applications of the surface operator to study of the phase properties of QCD. One of the possible explanations of the quark confinement is the condensation of magnetic monopoles in the vacuum [1] as dual superconductor models. The BCS theory of superconductivity explains superconductivity as the result of the condensation of electric chargers to cooper pairs. In this case a string occurs between monopoles. In a dual superconductor an analogous effect occurs through the condensation of magnetic charges (also called magnetic monopoles), and a string connects electrically charged particles. According to 't Hooft [2], monopoles can appear as a result of partial break of gauge symmetry. In this work we break $S U(2)$ symmetry saving $U(1)$ group symmetry.

A surface operator [3] is sensitive to existence of monopoles. It was first introduced by Witten $[4,5]$. It is determined by the divergence of the chromomagnetic field through a closed surface. We use the lattice calculations to study the structure of the vacuum in $S U(2)$ gluodynamics.

In non-abelian pure gauge theories, the expectation values of large surface operators are difficult to compute via numerical simulation because the increase of surface area makes the signalto-noise ratio decay very fast. We adapt the multilevel scheme [6] introduced for line operators to work with surface operators when area exceeds $1 \mathrm{fm}^{2}$.

\section{Witten parameter}

We consider the following definition of the Witten parameter on the lattice:

$$
W_{p}(S)=R e \prod_{S} e^{i \theta_{p}},
$$

where $\theta_{p}$ is a plaquette angle. The $\theta_{p}$ relates to the $F_{\mu \nu}$ by the following relation:

$$
F_{p}=\widehat{1} \cos \theta_{p}+\imath n_{i} \sigma_{i} \sin \theta_{p},
$$

where $n_{i}$ is a vector on the unit sphere, $\sigma_{i}$ is the Pauli matrices, $F_{p}$ is a value of the gauge field tensor $F_{\mu v}$ on the plaquette. Then, for the $\theta_{p}$ we can write the following definition:

$$
\theta_{p}=\arccos \left(\frac{1}{2} \operatorname{Tr} F_{p}\right) .
$$

All the phases are calculated on the surface of a cube in the four-dimensional space-time. The range of function $\arccos (x)$ is $[0, \pi]$. In the gauge group $U(1)$ the range of the angle is $[0,2 \pi]$. Hence, on one side of the cube the phase is selected as $+\arccos \left(\frac{1}{2} \operatorname{Tr} F_{p}\right)$, whereas on the opposite side it is $-\arccos \left(\frac{1}{2} \operatorname{Tr} F_{p}\right)$. 

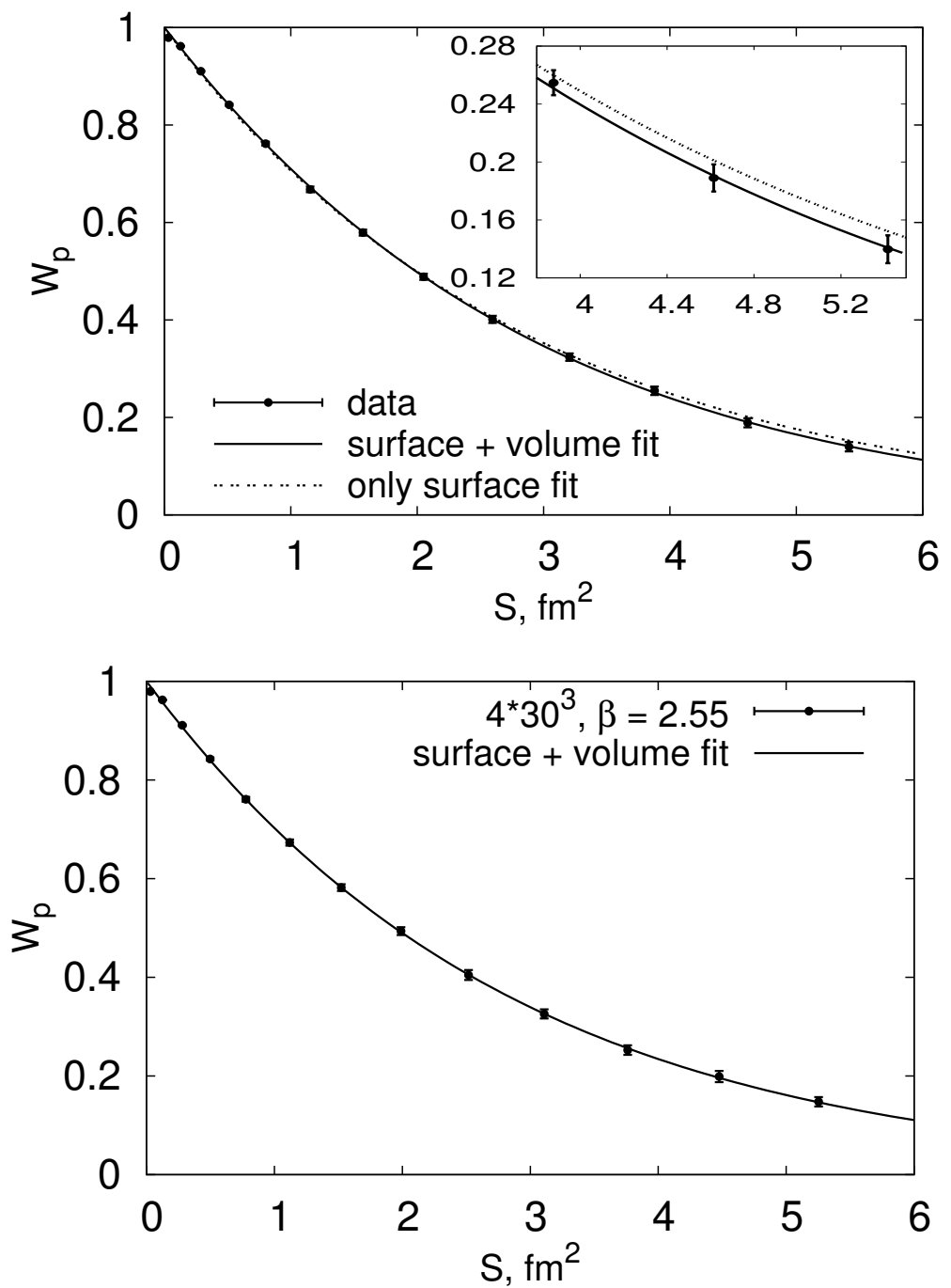

Figure 1: Dependence of the Witten parameter on a surface area in the confinement phase (up panel) and comparison of fittings. Lattice size is $41^{4}$ and $\beta=2.55$. The same can be observed in the deconfinement phase (bottom panel).

\section{Results}

All calculations are performed on 50 configurations in 1000 points on each lattice configuration. For both phases results are shown at the Figure 1. We can say that these two dependencies are the same. To understand better the behaviour of the Witten parameter we fit our dependence as follows

$$
W_{p}(S, V)=e^{-\sigma S-\gamma V},
$$

where $\sigma$ is a surface coefficient, $\gamma$ is a volume coefficient, $S$ is a surface area, $V$ is the cube volume. We use minuit2 library from ROOT package [10] to fit numerical results. The Figure 1 shows that the fitting is good if $\sigma, \gamma \neq 0$. Then in both phases the Witten parameter depends on the cube volume and surface area. 
In conclusion we can say that the Witten parameter depends on a surface area and volume in both phases. Consequently, the Witten parameter cannot be considered as the order parameter of confinement-deconfinement phase transition. This result is similar to the behaviour of spatial Wilson loops expectation value, which is not sensitive to the phase transition. It might be interesting to study surface operators to calculate the Witten parameter on the cube with two spatial axis and one temporal axis. Another important question that has to be clarified is the plaquette angle definition.

\section{Acknowledgments}

We are thankful to Dr. M. I. Polikarpov, Dr. P. V. Buividovich, Dr. V. G. Bornyakov, Dr. V. I. Zakharov and Dr. V. V. Braguta for interesting and useful discussions. The authors are much obliged to Dr. A. M. Shirokov for useful comments. Numerical calculations were performed with the equipment of the Center for Collective Use "Far Eastern Computing Resource" (Vladivostok).

\section{References}

[1] M. N. Chernodub and M. I. Polikarpov, arXiv:hep-th/9710205 [hep-th] (1997).

[2] G. 't Hooft, Nuclear Physics B 190, 455 (1981).

[3] S. Gukov, "Phases of gauge theories and surface operators" the talk presented at the workshop "Prestrings", 11-15 August, 2008, Zürich, Switzerland.

[4] S. Gukov and E. Witten, arXiv:hep-th/0612073v2 [hep-th] (2007).

[5] S. Gukov and E. Witten, arXiv:0804.1561 [hep-th] (2008).

[6] M. Lüscher and P. Weisz, JHEP 0109, 010 (2001), arXiv:hep-lat/0108014 [hep-lat].

[7] K. Wilson, Phys. Rev. D 10, 2445 (1974).

[8] M. Creutz, Quarks, gluons and lattice Cambridge University Press, Cambridge, 1983.

[9] G. Parisi, R. Petronzio and F. Rapuano, Physics Letters B 128, 418 (1983).

[10] The ROOT library, http://root.cern.ch/drupal/ .

[11] A. Di Giacomo and V. I. Zakharov, Phys. Atom. Nucl. 73, 711 (2010), arXiv:0806:2938 [hep-th]. 